\title{
A Physical Interpretation of Tight Frames
}

P. G. Casazza ${ }^{1}$, M. Fickus ${ }^{2}$, J. Kovačević ${ }^{3}$, M. T. Leon ${ }^{4}$, and J. C. Tremain ${ }^{5}$

1 Mathematics Department, University of Missouri, Columbia, Missouri 65211

USA, pete@math.missouri.edu

2 Department of Mathematics and Statistics, Air Force Institute of Technology, Wright-Patterson AFB, Ohio 45433 USA, Matthew.Fickus@afit.edu

3 Department of Biomedical Engineering, Carnegie Mellon University, Pittsburgh, Pennsylvania 15213, USA, jelenak@cmu.edu

4 Mathematics Department, University of Missouri, Columbia, Missouri 65211

USA, mleon@math.missouri.edu

5 Mathematics Department, University of Missouri, Columbia, Missouri 65211

USA, janet@math.missouri.edu

Summary. We find finite tight frames when the lengths of the frame elements are predetermined. In particular, we derive a "fundamental inequality" which completely characterizes those sequences which arise as the lengths of a tight frame's elements. Furthermore, using concepts from classical physics, we show that this characterization has an intuitive physical interpretation.

\section{Introduction}

Let $\mathbb{H}_{N}$ be a finite $N$-dimensional Hilbert space. A finite sequence $\left\{f_{m}\right\}_{m=1}^{M}$ of vectors is $A$-tight for $\mathbb{H}_{N}$ if there exists $A \geq 0$ such that,

$$
A\|f\|^{2}=\sum_{m=1}^{M}\left|\left\langle f, f_{m}\right\rangle\right|^{2},
$$

for all $f \in \mathbb{H}_{N}$. An $A$-tight frame is an $A$-tight sequence for which $A>0$. By polarization, $\left\{f_{m}\right\}_{m=1}^{M}$ is $A$-tight for $\mathbb{H}_{N}$ if and only if,

$$
A f=\sum_{m=1}^{M}\left\langle f, f_{m}\right\rangle f_{m},
$$

for all $f \in \mathbb{H}_{N}$. Clearly, any orthonormal basis is a 1-tight frame. However, the converse is false. For example, the vertices of a tetrahedron, appropriately centered and scaled, form a 1-tight frame of four elements for $\mathbb{R}^{3}$. Moreover, while the elements of an orthonormal basis are of unit length a priori, there are no explicit assumptions made about the lengths of a tight frame's elements. This raises the question, 
Given positive integers $M$ and $N$, for what sequences of nonnegative numbers $\left\{a_{m}\right\}_{m=1}^{M}$ do there exist tight frames $\left\{f_{m}\right\}_{m=1}^{M}$ for $\mathbb{H}_{N}$, such that $\left\|f_{m}\right\|=a_{m}$ for all $m$ ?

The answer to this question is the subject of this chapter. To be more precise, we first derive a necessary condition upon the lengths.

Proposition 1. If $\left\{f_{m}\right\}_{m=1}^{M}$ is A-tight for $\mathbb{H}_{N}$ then,

$$
\max _{m=1, \ldots, M}\left\|f_{m}\right\|^{2} \leq A=\frac{1}{N} \sum_{m=1}^{M}\left\|f_{m}\right\|^{2} .
$$

Proof. First note that for any $m=1, \ldots, M$,

$$
\left\|f_{m}\right\|^{4}=\left|\left\langle f_{m}, f_{m}\right\rangle\right|^{2} \leq \sum_{m^{\prime}=1}^{M}\left|\left\langle f_{m}, f_{m^{\prime}}\right\rangle\right|^{2}=A\left\|f_{m}\right\|^{2} .
$$

Thus, $\left\|f_{m}\right\|^{2} \leq A$ for all $m=1, \ldots, M$, yielding the inequality in (1). For the equality, let $\left\{e_{n}\right\}_{n=1}^{N}$ be an orthonormal basis for $\mathbb{H}_{N}$. By Parseval's identity,

$$
\frac{1}{N} \sum_{m=1}^{M}\left\|f_{m}\right\|^{2}=\frac{1}{N} \sum_{m=1}^{M} \sum_{n=1}^{N}\left|\left\langle f_{m}, e_{n}\right\rangle\right|^{2}=\frac{1}{N} \sum_{n=1}^{N} A\left\|e_{n}\right\|^{2}=A .
$$

Thus, the lengths $\left\{a_{m}\right\}_{m=1}^{M}$ of a tight frame of $M$ elements for an $N$ dimensional space must satisfy the fundamental inequality,

$$
\max _{m=1, \ldots, M} a_{m}^{2} \leq \frac{1}{N} \sum_{m=1}^{M} a_{m}^{2}
$$

Remarkably, this easily-found necessary condition will prove sufficient as well. That is, we shall show that for any sequence $\left\{a_{m}\right\}_{m=1}^{M}$ which satisfies (2), and for any $N$-dimensional Hilbert space $\mathbb{H}_{N}$, there exists a tight frame $\left\{f_{m}\right\}_{m=1}^{M}$ for $\mathbb{H}_{N}$ for which $\left\|f_{m}\right\|=a_{m}$ for all $m$.

Furthermore, when given a sequence $\left\{a_{m}\right\}_{m=1}^{M}$ which violates (2), we shall determine those sequences $\left\{f_{m}\right\}_{m=1}^{M}$ of norms $\left\{a_{m}\right\}_{m=1}^{M}$ which are as close as possible to being tight frames, in a natural, intuitive sense.

Of course, this problem does not exist in a vacuum. Frames have been a subject of interest for some time, both in theory and in applications. In recent years, several inquiries have been made into some of the deeper issues of finite tight frames.

The theory of frames was first introduced by Duffin and Schaeffer [10] in the 1950's, furthering the study of nonharmonic Fourier series and the time-frequency decompositions of Gabor [13]. Decades later, the subject was reinvigorated following a publication of Daubechies, Grossman and Meyer [9]. Frames have subsequently become a state-of-the-art signal processing tool. 
Frames provide redundant vector space decompositions, which are very attractive from the applied perspective. In particular, frame decompositions are resilient against noise and quantization, and provide numerically stable reconstruction algorithms $[6,8,15]$. Translation-invariant frames in $\ell^{2}(\mathbb{Z})$, being equivalent to perfect reconstruction oversampled filter banks, have been studied extensively $[4,7,18,19]$. Frame decompositions may also reveal hidden signal characteristics, and have therefore been used to solve problems of detection $[1,3,21]$. Frames have also been used to design unitary space-time constellations for multiple-antenna wireless systems [16].

Specific types of finite tight frames have been studied to solve problems in communications $[14,17,20,24]$. In addition, many techniques of constructing finite tight frames have been discovered, several of which involve group theory $[5,22]$. Researchers have also been interested in tight frames whose elements are restricted to spheres and ellipsoids $[2,11]$, as well as the manifold structures of spaces of all such frames [12].

Much of our work is inspired by Benedetto and Fickus's characterization of unit-norm tight frames [2]. Subsequent to the completion of our work, we learned that some of our results were independently obtained by Anantharam and Viswanath [23] in the context of wireless communications. At the conclusion of this chapter, we compare and contrast the two approaches.

In the following section, we motivate our main results by introducing a physical interpretation of frame theory, extending the frame-equivalent notions of force and potential energy first introduced by Benedetto and Fickus. Section 3 contains several results concerning the minimization of this generalized frame potential, highlighting the connection between optimal energy and tightness. Finally, in Section 4, we characterize the lengths of a tight frame's elements in terms of the fundamental inequality, and discuss this characterization from the physical perspective.

\section{The Physical Theory}

Given any nonnegative sequence $\left\{a_{m}\right\}_{m=1}^{M}$ which satisfies the fundamental inequality (2), our goal is to construct a tight frame $\left\{f_{m}\right\}_{m=1}^{M}$ for $\mathbb{H}_{N}$ such that $\left\|f_{m}\right\|=a_{m}$ for all $m$. We begin by briefly discussing the special case of this problem when $a_{m}=1$ for all $m$.

A unit-norm tight frame (UNTF) is a tight frame whose frame elements are normalized. In the past few years, several independent proofs have been found which show that such frames always exist, that is, for any $N$-dimensional Hilbert space $\mathbb{H}_{N}$, and any $M \geq N$, there exists a tight frame of $M$ elements $\left\{f_{m}\right\}_{m=1}^{M}$ for $\mathbb{H}_{N}$ such that $\left\|f_{m}\right\|=1$ for all $m$. One proof involves an explicit construction of such frames using orthogonal projections of finite Fourier bases. However, the construction does not generalize to the case of nonuniform lengths. 
Another proof is given by Benedetto and Fickus [2], who then referred to unit-norm tight frames as normalized tight frames. Their approach was more qualitative in nature, being inspired by the high level of symmetry in many canonical examples of UNTFs. Specifically, they found a connection between UNTFs and a classical means of equally distributing points on a sphere. Using this perspective, Benedetto and Fickus characterized UNTFs as the minimizers of a potential energy function, thus guaranteeing their existence.

In this section, we show how Benedetto and Fickus's notions of "frame force" and "frame potential" may be extended to the nonuniform setting. Inspired by the Coulomb force, consider the frame force $\mathrm{FF}\left(f_{m}, f_{m^{\prime}}\right) \in \mathbb{R}^{N}$ of $f_{m^{\prime}} \in \mathbb{R}^{N}$ upon $f_{m} \in \mathbb{R}^{N}$,

$$
\mathrm{FF}\left(f_{m}, f_{m^{\prime}}\right)=2\left\langle f_{m}, f_{m^{\prime}}\right\rangle\left(f_{m}-f_{m^{\prime}}\right) .
$$

This is essentially the same definition that appears in [2], but without the restriction of $f_{m}$ and $f_{m^{\prime}}$ to the unit sphere. Compared to the forces of the natural world, the frame force is bizarre. For example, this force is not translationinvariant. Also, the force between coincidental points is well-defined, being zero. Most importantly, the frame force "encourages orthogonality" — the force is repulsive when the angle between two vectors is acute, attractive when this angle is obtuse, and zero when the vectors are perpendicular.

Now consider a physical system of $M$ points $\left\{f_{m}\right\}_{m=1}^{M}$ whose movement is restricted to concentric spheres about the origin of radii $\left\{a_{m}\right\}_{m=1}^{M}$, respectively, in which each point pushes against all the others, according to the frame force. Dynamically, each point tries to force the others into perpendicularity. At the same time, when $M>N$, it is impossible for $\left\{f_{m}\right\}_{m=1}^{M} \subset \mathbb{R}^{N}$ to consist of mutually orthogonal vectors. Nevertheless, given enough time and even the smallest amount of friction, the points will eventually settle into an equilibrium. The result is a sequence of vectors $\left\{f_{m}\right\}_{m=1}^{M}$, with $\left\|f_{m}\right\|=a_{m}$ for all $m$, which are "as close to being mutually orthogonal as possible."

Formally, equilibria are characterized as the local minimizers of the potential energy function. For a system of particles pushing against each other according to the frame force, this energy function is known as the "frame potential." To be precise, for $a \geq 0$, let $\mathrm{S}(a)$ be the hypersphere $\left\{f \in \mathbb{R}^{N}:\|f\|=a\right\}$. Similarly, for any nonnegative sequence $\left\{a_{m}\right\}_{m=1}^{M}$ let,

$$
\mathrm{S}\left(\left\{a_{m}\right\}_{m=1}^{M}\right)=\mathrm{S}\left(a_{1}\right) \times \cdots \times \mathrm{S}\left(a_{M}\right) .
$$

The frame potential is a function FP : $\mathrm{S}\left(\left\{a_{m}\right\}_{m=1}^{M}\right) \rightarrow \mathbb{R}$, such that for any $\left\{f_{m}\right\},\left\{g_{m}\right\} \in \mathrm{S}\left(\left\{a_{m}\right\}_{m=1}^{M}\right)$, their difference in potentials represents the work required to transform $\left\{g_{m}\right\}$ into $\left\{f_{m}\right\}$, under the influence of the frame force. The following result provides an explicit form of this potential.

Proposition 2. For any $\left\{f_{m}\right\} \in \mathrm{S}\left(\left\{a_{m}\right\}_{m=1}^{M}\right)$,

$$
\operatorname{FP}\left(\left\{f_{m}\right\}\right)=\sum_{m=1}^{M} \sum_{m^{\prime}=1}^{M}\left|\left\langle f_{m}, f_{m^{\prime}}\right\rangle\right|^{2} .
$$


Proof. Recall $f_{m} \in \mathrm{S}\left(a_{m}\right) \subset \mathbb{R}^{N}$ for all $m$. Thus, for any $m, m^{\prime}=1, \ldots, M$,

$$
\left\|f_{m}-f_{m^{\prime}}\right\|^{2}=a_{m}^{2}-2\left\langle f_{m}, f_{m^{\prime}}\right\rangle+a_{m^{\prime}}^{2} .
$$

Solving for $\left\langle f_{m}, f_{m^{\prime}}\right\rangle$ in (4) and substituting this expression in (3), we see that frame force may be written completely in terms of $f_{m}-f_{m^{\prime}}$,

$$
\mathrm{FF}\left(f_{m}, f_{m^{\prime}}\right)=\left(a_{m}^{2}+a_{m^{\prime}}^{2}-\left\|f_{m}-f_{m^{\prime}}\right\|^{2}\right)\left(f_{m}-f_{m^{\prime}}\right) .
$$

As this field is conservative, the potential energy is computed using an antigradient. This is accomplished by first anti-differentiating the "scalar force,"

$$
-\int\left(a_{m}^{2}+a_{m^{\prime}}^{2}-x^{2}\right) x d x=\frac{1}{4} x^{2}\left[x^{2}-2\left(a_{m}^{2}+a_{m^{\prime}}^{2}\right)\right],
$$

and then evaluating at $x=\left\|f_{m}-f_{m^{\prime}}\right\|$,

$$
\frac{1}{4}\left\|f_{m}-f_{m^{\prime}}\right\|^{2}\left[\left\|f_{m}-f_{m^{\prime}}\right\|^{2}-2\left(a_{m}^{2}+a_{m^{\prime}}^{2}\right)\right]=\left\langle f_{m}, f_{m^{\prime}}\right\rangle^{2}-\frac{1}{4}\left(a_{m}^{2}+a_{m^{\prime}}^{2}\right)^{2},
$$

which is simplified using (4). This quantity represents the frame potential energy at $f_{m}$ from the field generated by $f_{m^{\prime}}$. The total energy is the sum of these pairwise potentials,

$$
\sum_{m=1}^{M} \sum_{m^{\prime} \neq m}\left[\left\langle f_{m}, f_{m^{\prime}}\right\rangle^{2}-\frac{1}{4}\left(a_{m}^{2}+a_{m^{\prime}}^{2}\right)^{2}\right] .
$$

As potential energy is defined in terms of differences, this function is only unique up to additive constants. We choose to omit the terms $\left(a_{m}^{2}+a_{m^{\prime}}^{2}\right)^{2} / 4$, and include the diagonal terms $\left|\left\langle f_{m}, f_{m}\right\rangle\right|^{2}=a_{m}^{4}$, yielding the result.

In the following sections, we shall use this expression of the frame potential FP $: \mathrm{S}\left(\left\{a_{m}\right\}_{m=1}^{M}\right) \rightarrow \mathbb{R}$ to characterize tight frames of lengths $\left\{a_{m}\right\}_{m=1}^{M}$. Specifically, we characterize such frames in terms of minimizers of FP, that is, in terms of those "maximally orthogonal" sequences $\left\{f_{m}\right\} \in \mathrm{S}\left(\left\{a_{m}\right\}_{m=1}^{M}\right)$ which are in equilibrium with respect to the frame force.

Before continuing, we pause to consider another physical aspect of the frame force (3), namely that the power of a frame force field generated by $f_{m^{\prime}}$ will increase with $\left\|f_{m^{\prime}}\right\|$. That is, points which are farther away from the origin will apply a stronger push than those which are closer.

To determine the explicit dependence, consider the effective component of the frame force $\mathrm{FF}\left(f_{m}, f_{m^{\prime}}\right)$ which lies parallel to the surface of $\mathrm{S}\left(a_{m}\right)$ at $f_{m}$. In essence, the effective component is the only part of the frame force that a point will actually experience, as the point is prohibited from moving in the direction of the normal component. Formally, the effective component is, 


$$
\begin{aligned}
\operatorname{EFF}\left(f_{m}, f_{m^{\prime}}\right) & =\mathrm{FF}\left(f_{m}, f_{m^{\prime}}\right)-\operatorname{Proj}_{f_{m}} \mathrm{FF}\left(f_{m}, f_{m^{\prime}}\right), \\
& =\mathrm{FF}\left(f_{m}, f_{m^{\prime}}\right)-\frac{\left\langle\mathrm{FF}\left(f_{m}, f_{m^{\prime}}\right), f_{m}\right\rangle}{\left\|f_{m}\right\|^{2}} f_{m}, \\
& =\frac{2\left|\left\langle f_{m}, f_{m^{\prime}}\right\rangle\right|^{2}}{a_{m}^{2}} f_{m}-2\left\langle f_{m}, f_{m^{\prime}}\right\rangle f_{m^{\prime}},
\end{aligned}
$$

for $f_{m} \neq 0$. Clearly, $\left\|\operatorname{EFF}\left(f_{m}, f_{m^{\prime}}\right)\right\|$ grows as a square of $\left\|f_{m^{\prime}}\right\|$. We therefore refer to $a_{m}^{2}$ as the power of $f_{m}$. The fundamental inequality (2) may therefore be interpreted as requiring a somewhat uniform distribution of power. Conversely, when the fundamental inequality is violated, then a single mass $a_{m^{\prime}}$ is disproportionately large, and it is therefore conceivable that a state of equilibrium is only achieved when the massive point $f_{m^{\prime}}$ has forced the remaining points into perpendicularity. In the final section, we shall show that this is indeed the case, and further elaborate upon these phenomena.

\section{The Physical Interpretation of Frames}

Above, we discussed how the frame force may be used to define an intuitive notion of a "maximally orthogonal" sequence of vectors. We now go beyond intuition, and establish a rigorous link between the physical theory and the theory of frames. Our work makes repeated use of the canonical linear operators of frame theory. In particular, given $\left\{f_{m}\right\}_{m=1}^{M} \subset \mathbb{H}_{N}$, consider the analysis operator $F: \mathbb{H}_{N} \rightarrow \mathbb{C}^{M}$,

$$
(F f)(m)=\left\langle f, f_{m}\right\rangle,
$$

whose adjoint is the synthesis operator $F^{*}: \mathbb{C}^{M} \rightarrow \mathbb{H}_{N}$,

$$
F^{*} g=\sum_{m=1}^{M} g(m) f_{m} .
$$

Their compositions are the frame operator $F^{*} F: \mathbb{H}_{N} \rightarrow \mathbb{H}_{N}$,

$$
F^{*} F f=\sum_{m=1}^{M}\left\langle f, f_{m}\right\rangle f_{m},
$$

and the Gram matrix $F F^{*}: \mathbb{C}^{M} \rightarrow \mathbb{C}^{M}$, whose $\left(m, m^{\prime}\right)$ th matrix entry is $\left\langle f_{m^{\prime}}, f_{m}\right\rangle$. We note that $\left\{f_{m}\right\}_{m=1}^{M}$ is $A$-tight for $\mathbb{H}_{N}$ if and only if the corresponding analysis operator satisfies $\|F f\|^{2}=A\|f\|^{2}$ for all $f \in \mathbb{H}_{N}$, which is in turn equivalent to the frame operator satisfying $F^{*} F=A I$. In the following result, we use this equivalence to characterize tight frames in terms of the frame force. 
Proposition 3. $\left\{f_{m}\right\}_{m=1}^{M}$ is tight for $\mathbb{R}^{N}$ if and only if the effective force field generated by $\left\{f_{m}\right\}_{m=1}^{M}$ vanishes everywhere, that is, if and only if,

$$
\sum_{m=1}^{M} \operatorname{EFF}\left(f, f_{m}\right)=0
$$

for all $f \in \mathbb{R}^{N}$.

Proof. Let $F$ be the analysis operator of $\left\{f_{m}\right\}_{m=1}^{M}$. Then,

$$
0=\sum_{m=1}^{M} \operatorname{EFF}\left(f, f_{m}\right)=\sum_{m=1}^{M}\left[\frac{2\left|\left\langle f, f_{m}\right\rangle\right|^{2}}{\|f\|^{2}} f-2\left\langle f, f_{m}\right\rangle f_{m}\right],
$$

for all $f \in \mathbb{R}^{N}$ if and only if,

$$
F^{*} F f=\sum_{m=1}^{M}\left\langle f, f_{m}\right\rangle f_{m}=\sum_{m=1}^{M} \frac{\left|\left\langle f, f_{m}\right\rangle\right|^{2}}{\|f\|^{2}} f=\frac{\|F f\|^{2}}{\|f\|^{2}} f,
$$

for all $f \neq 0 \in \mathbb{R}^{N}$. Clearly (5) holds if $\left\{f_{m}\right\}_{m=1}^{M}$ is tight. For the converse, note that if (5) holds, then every $f \neq 0 \in \mathbb{R}^{N}$ is an eigenvector of $F^{*} F$. Thus, $F^{*} F=A I$ for some $A \geq 0$.

In light of this result, it is natural to ask whether every real tight frame is in equilibrium with respect to the frame force. Below, we answer this question in the affirmative by showing that every tight frame for $\mathbb{R}^{N}$ is a global minimizer of the frame potential.

Before continuing, note that one may "minimize the frame potential" in a more general setting. That is, despite being derived in the context of real Euclidean spaces, the formula for the frame potential makes sense in a general Hilbert space. To be precise, given a possibly complex finite-dimensional Hilbert space $\mathbb{H}_{N}$, consider the generalized frame potential,

$$
\mathrm{FP}: \mathbb{H}_{N}^{M} \rightarrow \mathbb{R}, \quad \operatorname{FP}\left(\left\{f_{m}\right\}\right)=\sum_{m=1}^{M} \sum_{m^{\prime}=1}^{M}\left|\left\langle f_{m}, f_{m^{\prime}}\right\rangle\right|^{2} .
$$

Often, we shall restrict the domain of FP to the Cartesian product $\mathrm{S}\left(\left\{a_{m}\right\}_{m=1}^{M}\right)$ of the $M$ generalized spheres $\mathrm{S}\left(a_{m}\right)=\left\{f \in \mathbb{H}_{N}:\|f\|=a_{m}\right\}$. In particular, the following results characterize tight frames of lengths $\left\{a_{m}\right\}_{m=1}^{M}$ as the global minimizers of this restricted potential. We begin by showing that the frame potential of a sequence is equal to the square of the Hilbert-Schmidt (Frobenius) norm of the corresponding frame operator.

Lemma 1. Let $F$ be the analysis operator of $\left\{f_{m}\right\}_{m=1}^{M} \subset \mathbb{H}_{N}$. Then,

$$
\operatorname{FP}\left(\left\{f_{m}\right\}_{m=1}^{M}\right)=\operatorname{Tr}\left(\left(F^{*} F\right)^{2}\right) .
$$


Proof. Let $\left\{e_{n}\right\}_{n=1}^{N}$ be an orthonormal basis of $\mathbb{H}_{N}$. Then,

$$
\begin{aligned}
\mathrm{FP}\left(\left\{f_{m}\right\}_{m=1}^{M}\right) & =\sum_{m=1}^{M} \sum_{m^{\prime}=1}^{M}\left\langle f_{m^{\prime}}, f_{m}\right\rangle\left\langle f_{m}, f_{m^{\prime}}\right\rangle, \\
& =\sum_{m=1}^{M} \sum_{m^{\prime}=1}^{M}\left\langle\sum_{n=1}^{N}\left\langle f_{m^{\prime}}, e_{n}\right\rangle e_{n}, f_{m}\right\rangle\left\langle f_{m}, f_{m^{\prime}}\right\rangle, \\
& =\sum_{n=1}^{N} \sum_{m=1}^{M} \sum_{m^{\prime}=1}^{M}\left\langle\left\langle e_{n}, f_{m}\right\rangle f_{m},\left\langle e_{n}, f_{m^{\prime}}\right\rangle f_{m^{\prime}}\right\rangle, \\
& =\sum_{n=1}^{N}\left\langle F^{*} F e_{n}, F^{*} F e_{n}\right\rangle, \\
& =\sum_{n=1}^{N}\left\langle\left(F^{*} F\right)^{2} e_{n}, e_{n}\right\rangle=\operatorname{Tr}\left(\left(F^{*} F\right)^{2}\right) .
\end{aligned}
$$

Proposition 4. For any $N$-dimensional Hilbert space $\mathbb{H}_{N}$ and any nonnegative sequence $\left\{a_{m}\right\}_{m=1}^{M}$, the frame potential $\mathrm{FP}: \mathrm{S}\left(\left\{a_{m}\right\}_{m=1}^{M}\right) \rightarrow \mathbb{R}$ satisfies,

$$
\frac{1}{N}\left[\sum_{m=1}^{M} a_{m}^{2}\right]^{2} \leq \mathrm{FP}\left(\left\{f_{m}\right\}\right) .
$$

Furthermore, this lower bound is achieved if and only if $\left\{f_{m}\right\}_{m=1}^{M}$ is tight for $\mathbb{H}_{N}$ with $\left\|f_{m}\right\|=a_{m}$ for all $m$.

Proof. Let $F$ be the analysis operator of $\left\{f_{m}\right\} \in \mathrm{S}\left(\left\{a_{m}\right\}_{m=1}^{M}\right)$, and let $\left\{\lambda_{n}\right\}_{n=1}^{N}$ be the eigenvalues of $F^{*} F$, counting multiplicities. By Lemma 1,

$$
\operatorname{FP}\left(\left\{f_{m}\right\}\right)=\operatorname{Tr}\left(F^{*} F\right)^{2}=\sum_{n=1}^{N} \lambda_{n}^{2}
$$

Meanwhile, the trace of the frame operator satisfies,

$$
\begin{aligned}
& \sum_{n=1}^{N} \lambda_{n}=\operatorname{Tr}\left(F^{*} F\right)=\sum_{n=1}^{N}\left\langle F^{*} F e_{n}, e_{n}\right\rangle=\sum_{n=1}^{N}\left\langle\sum_{m=1}^{M}\left\langle e_{n}, f_{m}\right\rangle f_{m}, e_{n}\right\rangle \\
&=\sum_{m=1}^{M} \sum_{n=1}^{N}\left|\left\langle f_{m}, e_{n}\right\rangle\right|^{2}=\sum_{m=1}^{M}\left\|f_{m}\right\|^{2}=\sum_{m=1}^{M} a_{m}^{2},
\end{aligned}
$$

where $\left\{e_{n}\right\}_{n=1}^{N}$ is any orthonormal basis for $\mathbb{H}_{N}$. The lower bound is therefore found by solving the constrained minimization problem,

$$
\min \left\{\sum_{n=1}^{N} \lambda_{n}^{2}: \sum_{n=1}^{N} \lambda_{n}=\sum_{m=1}^{M} a_{m}^{2}\right\} .
$$


Using Lagrange multipliers, the minimum is found to occur precisely when,

$$
\lambda_{1}=\cdots=\lambda_{N}=\frac{1}{N} \sum_{m=1}^{M} a_{m}^{2} .
$$

Thus, for any $\left\{f_{m}\right\} \in \mathrm{S}\left(\left\{a_{m}\right\}_{m=1}^{M}\right)$,

$$
\operatorname{FP}\left(\left\{f_{m}\right\}\right)=\sum_{n=1}^{N} \lambda_{n}^{2} \geq \sum_{n=1}^{N}\left[\frac{1}{N} \sum_{m=1}^{M} a_{m}^{2}\right]^{2}=\frac{1}{N}\left[\sum_{m=1}^{M} a_{m}^{2}\right]^{2} .
$$

Furthermore, this lower bound is achieved precisely when all the eigenvalues of $F^{*} F$ are equal, that is, when $F^{*} F=A I$ for some $A \geq 0$.

Note that the previous result does not imply that this lower bound on the frame potential is optimal. Rather, this bound is only achieved when there exists a tight frame $\left\{f_{m}\right\}_{m=1}^{M}$ for $\mathbb{H}_{N}$ with $\left\|f_{m}\right\|=a_{m}$ for all $m$. We emphasize that this result does not show that such frames actually exist. Indeed, such frames cannot exist when the requisite lengths $\left\{a_{m}\right\}_{m=1}^{M}$ violate the fundamental inequality.

These ambiguities will be resolved by the main results of the following section. In particular, we show that there always exists a tight frame of lengths $\left\{a_{m}\right\}_{m=1}^{M}$, provided $\left\{a_{m}\right\}_{m=1}^{M}$ satisfies the fundamental inequality, and, in the case when the inequality is violated, we determine the true minimum value of the frame potential.

We conclude this section by briefly discussing the minimization of the frame potential in another context. Two sequences $\left\{f_{m}\right\}_{m=1}^{M},\left\{g_{m}\right\}_{m=1}^{M} \subset \mathbb{H}_{N}$ are dual frames if their analysis operators satisfy $G^{*} F=I$, that is, if,

$$
f=\sum_{m=1}^{M}\left\langle f, f_{m}\right\rangle g_{m}
$$

for all $f \in \mathbb{H}_{N}$. Any spanning set $\left\{f_{m}\right\}_{m=1}^{M} \subset \mathbb{H}_{N}$ has at least one dual frame, namely the canonical dual $\left\{\widetilde{f}_{m}\right\}_{m=1}^{M} \equiv\left\{\left(F^{*} F\right)^{-1} f_{m}\right\}_{m=1}^{M}$ whose synthesis operator $\left(F^{*} F\right)^{-1} F$ is the pseudoinverse of $F$. Furthermore, when $M>N$, a sequence $\left\{f_{m}\right\}_{m=1}^{M} \subset \mathbb{H}_{N}$ may have an infinite number of dual frames. Nevertheless, the canonical dual has been found to be the "optimal" dual in certain applications [15]. The following result shows that the canonical dual is also the optimal dual from the point of view of the frame potential.

Proposition 5. Let $\left\{f_{m}\right\}_{m=1}^{M} \subset \mathbb{H}_{N}$ be a spanning set. Then, the canonical dual of $\left\{f_{m}\right\}_{m=1}^{M}$ is the unique dual of minimal frame potential.

Proof. Let $F, \widetilde{F}$ and $G$ denote the analysis operators of $\left\{f_{m}\right\}_{m=1}^{M}$, its canonical dual, and any arbitrary dual frame, respectively. Note that,

$$
G^{*} G=[\widetilde{F}+(G-\widetilde{F})]^{*}[\widetilde{F}+(G-\widetilde{F})]=\widetilde{F}^{*} \widetilde{F}+(G-\widetilde{F})^{*}(G-\widetilde{F}),
$$


as the middle terms vanish,

$$
(G-\widetilde{F})^{*} \widetilde{F}=G^{*} F\left(F^{*} F\right)^{-1}-\left(F^{*} F\right)^{-1} F^{*} F\left(F^{*} F\right)^{-1}=0 .
$$

By Lemma 1 , and the fact that $(G-\widetilde{F})^{*}(G-\widetilde{F})$ is positive semidefinite,

$$
\begin{aligned}
\operatorname{FP}\left(\left\{g_{m}\right\}\right) & =\operatorname{Tr}\left(G^{*} G\right)^{2}=\operatorname{Tr}\left[\widetilde{F}^{*} \widetilde{F}+(G-\widetilde{F})^{*}(G-\widetilde{F})\right]^{2}, \\
& \geq \operatorname{Tr}\left(\widetilde{F}^{*} \widetilde{F}^{2}\right)=\operatorname{FP}\left(\left\{\widetilde{f}_{m}\right\}\right),
\end{aligned}
$$

with equality if and only if $G=\widetilde{F}$.

Having found the dual of minimal energy, we next characterize those sequences for which the sum of their energy and their dual's energy is minimal.

Proposition 6. A spanning set $\left\{f_{m}\right\}_{m=1}^{M} \subset \mathbb{H}_{N}$ is a minimizer of,

$$
\operatorname{FP}\left(\left\{f_{m}\right\}_{m=1}^{M}\right)+\operatorname{FP}\left(\left\{\tilde{f}_{m}\right\}_{m=1}^{M}\right)
$$

if and only if $\left\{f_{m}\right\}_{m=1}^{M}$ is a 1-tight frame for $\mathbb{H}_{N}$.

Proof. Let $\left\{f_{m}\right\}_{m=1}^{M} \subset \mathbb{H}_{N}$ be a spanning set, and let $\left\{\lambda_{n}\right\}_{n=1}^{N}$ be the eigenvalues of the corresponding frame operator $F^{*} F$, counting multiplicities. As the frame operator of the canonical dual is $\left(F^{*} F\right)^{-1}$, Lemma 1 gives,

$$
\operatorname{FP}\left(\left\{f_{m}\right\}\right)+\operatorname{FP}\left(\left\{\widetilde{f}_{m}\right\}\right)=\operatorname{Tr}\left(\left(F^{*} F\right)^{2}\right)+\operatorname{Tr}\left(\left(F^{*} F\right)^{-2}\right)=\sum_{n=1}^{N}\left(\lambda_{n}^{2}+\lambda_{n}^{-2}\right) .
$$

For any $n, \lambda_{n}^{2}+1 / \lambda_{n}^{2}$ is minimized by letting $\lambda_{n}=1$. Thus, (6) is bounded below by $2 N$, and this lower bound is achieved if and only if $F^{*} F=I$, that is, if and only if $\left\{f_{m}\right\}_{m=1}^{M} \subset \mathbb{H}_{N}$ is a 1-tight frame for $\mathbb{H}_{N}$. By letting $\left\{f_{m}\right\}_{m=1}^{M}$ be the union of orthonormal bases with the required number of zero vectors, we see that such frames exist. Thus, the lower bound is indeed a minimum.

\section{The Fundamental Inequality}

In the first section, we showed that if there exists a tight frame $\left\{f_{m}\right\}_{m=1}^{M}$ for $\mathbb{H}_{N}$ with $\left\|f_{m}\right\|=a_{m}$ for all $m$, then the fundamental inequality,

$$
\max _{m=1, \ldots, M} a_{m}^{2} \leq \frac{1}{N} \sum_{m=1}^{M} a_{m}^{2}
$$

is satisfied. Furthermore, in the previous section, we showed that if such frames exist, then they are minimizers of the frame potential FP $: \mathrm{S}\left(\left\{a_{m}\right\}_{m=1}^{M}\right) \rightarrow \mathbb{R}$. In this section, we prove that the converse of the first result is true, and demonstrate a partial converse of the second.

To begin, recall that the fundamental inequality may be interpreted as requiring the powers $\left\{a_{m}^{2}\right\}_{m=1}^{M}$ of a tight frame $\left\{f_{m}\right\} \in \mathrm{S}\left(\left\{a_{m}\right\}_{m=1}^{M}\right)$ to be somewhat uniform in distribution. For a more precise definition of a "uniform distribution" in this context, consider the following result. 
Lemma 2. For any sequence $\left\{c_{m}\right\}_{m=1}^{M} \subset \mathbb{R}$ with $c_{1} \geq \cdots \geq c_{M} \geq 0$, and for any positive integer $N$, there is a unique index $N_{0}$ with $1 \leq N_{0} \leq N$, such that the inequality,

$$
(N-n) c_{n}>\sum_{m=n+1}^{M} c_{m}
$$

holds for $1 \leq n<N_{0}$, while the opposite inequality,

$$
(N-n) c_{n} \leq \sum_{m=n+1}^{M} c_{m}
$$

holds for $N_{0} \leq n \leq N$.

Proof. We begin by pointing out an implicit assumption of this result, namely that if $M<N$, any summation over an empty set of indices is regarded as zero. Let $\mathcal{I}$ be the set of indices such that (7) holds. As $N \in \mathcal{I}$, then $\mathcal{I} \neq \emptyset$. Also, if $n \in \mathcal{I}$, then $n+1 \in \mathcal{I}$, since,

$$
\begin{aligned}
{[N-(n+1)] c_{n+1}=-c_{n+1}+(N-n) c_{n+1} } & \leq-c_{n+1}+(N-n) c_{n}, \\
& \leq-c_{n+1}+\sum_{m=n+1}^{N} c_{m}=\sum_{m=n+2}^{N} c_{m} .
\end{aligned}
$$

$N_{0}$ is therefore uniquely defined as the minimum index in $\mathcal{I}$.

Thus, for a given positive integer $N$, the index $N_{0}$ is the place in the sequence $\left\{c_{m}\right\}_{m=1}^{M}$ where the terms cease to be larger than the "average" of the smaller remaining terms. Of course, this is not a true average unless $M=N$. Nevertheless, one expects the index $N_{0}$ to be small if the sequence $\left\{c_{m}\right\}$ is somewhat evenly distributed, and large if $\left\{c_{m}\right\}$ varies greatly.

In the context of sequences $\left\{f_{m}\right\} \in \mathrm{S}\left(\left\{a_{m}\right\}_{m=1}^{M}\right) \subset \mathbb{H}_{N}^{M}$, we apply Lemma 2 to the sequence obtained by rearranging the powers $\left\{a_{m}^{2}\right\}_{m=1}^{M}$ in decreasing order. Let the irregularity of $\left\{a_{m}\right\}_{m=1}^{M}$ be $N_{0}-1$, where $N_{0}$ is the unique index obtained in this manner. The next result characterizes the fundamental inequality in terms of the irregularity.

Lemma 3. For a postive integer $N$, a nonnegative sequence $\left\{a_{m}\right\}_{m=1}^{M}$ satisfies the fundamental inequality if and only if the irregularity of $\left\{a_{m}\right\}_{m=1}^{M=1}$ is zero.

Proof. Without loss of generality, we assume $\left\{a_{m}\right\}_{m=1}^{M}$ is arranged in decreasing order. Thus, $\left\{a_{m}\right\}_{m=1}^{M}$ satisfies the fundamental inequality if and only if,

$$
(N-1) a_{1}^{2}=-a_{1}^{2}+N \max _{m} a_{m}^{2} \leq-a_{1}^{2}+\frac{N}{N} \sum_{m=1}^{M} a_{m}^{2}=\sum_{m=2}^{M} a_{m}^{2},
$$

that is, when the index obtained by applying Lemma 2 to $\left\{a_{m}^{2}\right\}_{m=1}^{M}$ is $N_{0}=1$, which is equivalent to an irregularity of $N_{0}-1=0$. 
Thus, for an arbitrary nonnegative sequence $\left\{a_{m}\right\}_{m=1}^{M}$, the irregularity serves to measure the degree to which the fundamental inequality is violated, and partitions the corresponding points $\left\{f_{m}\right\}_{m=1}^{M}$ into two camps, one strong and the other weak. This idea plays a key role in the following result, in which we completely characterize those sequences in equilibrium under the frame force.

Theorem 1. Let $\mathbb{H}_{N}$ be any $N$-dimensional Hilbert space, and let $\left\{a_{m}\right\}_{m=1}^{M}$ be a nonnegative decreasing sequence of irregularity $N_{0}-1$. Then, any local minimizer of the frame potential $\mathrm{FP}: \mathrm{S}\left(\left\{a_{m}\right\}_{m=1}^{M}\right) \rightarrow \mathbb{R}$ is of the form,

$$
\left\{f_{m}\right\}_{m=1}^{M}=\left\{f_{m}\right\}_{m=1}^{N_{0}-1} \cup\left\{f_{m}\right\}_{m=N_{0}}^{M},
$$

where $\left\{f_{m}\right\}_{m=1}^{N_{0}-1}$ is an orthogonal sequence for whose orthogonal complement the sequence $\left\{f_{m}\right\}_{m=N_{0}}^{M}$ is tight. Moreover, $\left\{f_{m}\right\}_{m=N_{0}}^{M}$ is a tight frame for this complement when the number of nonzero elements of $\left\{a_{m}\right\}_{m=1}^{M}$ is at least $N$.

Proof. Let $F$ be the analysis operator of a local minimizer $\left\{f_{m}\right\}$ of the frame potential FP $: \mathrm{S}\left(\left\{a_{m}\right\}_{m=1}^{M}\right) \rightarrow \mathbb{R}$. Let $\left\{\lambda_{j}\right\}_{j=1}^{J}$ be the nonnegative decreasing sequence of the distinct eigenvalues of $F^{*} F$, and let $\left\{E_{j}\right\}_{j=1}^{J}$ be the corresponding sequence of mutually orthogonal eigenspaces. Consider the sequence of indexing sets,

$$
\left\{\mathcal{I}_{j}\right\}_{j=1}^{J}, \quad \mathcal{I}_{j} \equiv\left\{m: F^{*} F f_{m}=\lambda_{j} f_{m}\right\} \equiv\left\{m: f_{m} \in E_{j}\right\} \subseteq\{1, \ldots, M\} .
$$

The remainder of the argument is outlined in the form of seven claims:

1. Each $f_{m}$ is an eigenvector for $F^{*} F$.

2. For any $j=1, \ldots, J,\left\{f_{m}\right\}_{m \in \mathcal{I}_{j}}$ is $\lambda_{j}$-tight for $E_{j}$.

3. For any $j<J,\left\{f_{m}\right\}_{m \in \mathcal{I}_{j}}$ is linearly independent.

4. For any $j<J,\left(1 / \sqrt{\lambda_{j}}\right)\left\{f_{m}\right\}_{m \in \mathcal{I}_{j}}$ is an orthonormal basis for $E_{j}$.

5. $\left(N-M+\left|\mathcal{I}_{J}\right|\right) \lambda_{J}=\sum_{m \in \mathcal{I}_{J}} a_{m}^{2}$.

6. $\left\{N_{0}, \ldots, M\right\} \subseteq \mathcal{I}_{J}$.

7. $\left\{N_{0}, \ldots, M\right\}=\mathcal{I}_{J}$.

Claim 1: Each $f_{m}$ is an eigenvector for $F^{*} F$. The proof of this claim is essentially the same as that of Theorem 7.3 in [2]. As such, we only provide a brief sketch of the argument. For any $m=1, \ldots, M$, consider the function obtained by allowing the $m$ th argument of the frame potential to vary, while holding the others constant at the minimizer $\left\{f_{m}\right\}$,

$$
\mathrm{FP}_{m}: \mathbb{H}_{N} \rightarrow \mathbb{R}, \quad \mathrm{FP}_{m}(f)=a_{m}^{4}+\sum_{m^{\prime} \neq m}\left|\left\langle f, f_{m^{\prime}}\right\rangle\right|^{2}+\mathrm{FP}\left(\left\{f_{m^{\prime}}\right\}_{m^{\prime} \neq m}\right) .
$$

Clearly, $f_{m}$ is a local minimizer of the constrained function $\mathrm{FP}_{m}: \mathrm{S}\left(a_{m}\right) \rightarrow \mathbb{R}$. Thus, there exists $c \in \mathbb{R}$ for which the corresponding Lagrange equation,

$$
\nabla \mathrm{FP}_{m}(f)=c \nabla\|f\|^{2},
$$


is satisfied at $f=f_{m}$. An explicit derivation of these gradients then reveals that $F^{*} F f_{m}=(1+c / 2) f_{m}$. Since $m$ is arbitrary, the claim is demonstrated.

As a consequence of the first claim, the elements of the minimizer $\left\{f_{m}\right\}_{m=1}^{M}$ are partitioned according to the eigenvalues. To be precise, we have,

$$
\bigcup_{j=1}^{J} \mathcal{I}_{j}=\{1, \ldots, M\}, \quad \mathcal{I}_{j} \cap \mathcal{I}_{j^{\prime}}=\emptyset \quad \forall j \neq j^{\prime},
$$

where, without loss of generality, we regard $m \in \mathcal{I}_{J}$ if $f_{m}=0$.

Claim 2: For any $j=1, \ldots, J,\left\{f_{m}\right\}_{m \in \mathcal{I}_{j}}$ is $\lambda_{j}$-tight for $E_{j}$. Fix $j=1, \ldots, J$, and let $F_{j}: E_{j} \rightarrow \mathbb{C}^{\left|\mathcal{I}_{j}\right|}$ be the anaylsis operator of $\left\{f_{m}\right\}_{m \in \mathcal{I}_{j}}$. Note that as the distinct eigenspaces of $F^{*} F$ are mutually orthogonal, then $\left\langle f, f_{m}\right\rangle=0$ for any $f \in E_{j}$ and any $m \notin \mathcal{I}_{j}$. Thus, for any $f \in E_{j}$,

$$
\lambda_{j} f=F^{*} F f=\sum_{m=1}^{M}\left\langle f, f_{m}\right\rangle f_{m}=\sum_{m \in \mathcal{I}_{j}}\left\langle f, f_{m}\right\rangle f_{m}=F_{j}^{*} F_{j} f,
$$

and so $F_{j}^{*} F_{j}: E_{j} \rightarrow E_{j}$ satisfies $F_{j}^{*} F_{j}=\lambda_{j} I$, yielding the claim.

Claim 3: For any $j<J,\left\{f_{m}\right\}_{m \in \mathcal{I}_{j}}$ is linearly independent. Assume to the contrary that $\left\{f_{m}\right\}_{m \in \mathcal{I}_{j}}$ is linearly dependent for some $j=1, \ldots, J-1$. We find a sequence of parametrized curves $\left\{g_{m}\right\}_{m=1}^{M}:(-1,1) \rightarrow \mathrm{S}\left(\left\{a_{m}\right\}_{m=1}^{M}\right)$, such that $\left\{g_{m}(0)\right\}_{m=1}^{M}=\left\{f_{m}\right\}_{m=1}^{M}$, and for which,

$$
\mathrm{FP}\left(\left\{g_{m}(t)\right\}_{m=1}^{M}\right)<\operatorname{FP}\left(\left\{f_{m}\right\}_{m=1}^{M}\right),
$$

for all $0 \neq t \in(-1,1)$, contradicting the global assumption that $\left\{f_{m}\right\}_{m=1}^{M}$ is a local minimizer of the frame potential. To begin, fix $h \in E_{J}$ with $\|h\|=1$. Since $\left\{f_{m}\right\}_{m \in \mathcal{I}_{j}}$ is linearly dependent, there exists a nonzero sequence of complex scalars $\left\{z_{m}\right\}_{m \in \mathcal{I}_{j}}$ such that $\left|z_{m}\right| \leq 1 / 2$ for all $m \in \mathcal{I}_{j}$, and for which,

$$
\sum_{m \in \mathcal{I}_{j}} \overline{z_{m}} a_{m} f_{m}=0
$$

For any $t \in(-1,1)$, consider $\left\{g_{m}(t)\right\}_{m=1}^{M} \subset \mathbb{H}_{N}$ given by,

$$
g_{m}(t)= \begin{cases}\sqrt{1-t^{2}\left|z_{m}\right|^{2}} f_{m}+t z_{m} a_{m} h, & m \in \mathcal{I}_{j}, \\ f_{m}, & m \notin \mathcal{I}_{j} .\end{cases}
$$

Clearly, $\left\{g_{m}(0)\right\}_{m=1}^{M}=\left\{f_{m}\right\}_{m=1}^{M}$. We now derive a Taylor approximation of $\operatorname{FP}\left(\left\{g_{m}(\cdot)\right\}_{m=1}^{M}\right):(-1,1) \rightarrow \mathbb{R}$ around $t=0$. By the product rule, 


$$
\begin{aligned}
& \frac{\mathrm{d}}{\mathrm{d} t} \mathrm{FP}\left(\left\{g_{m}(t)\right\}\right)=4 \operatorname{Re} \sum_{m=1}^{M} \sum_{m^{\prime}=1}^{M}\left\langle g_{m}^{\prime}, g_{m^{\prime}}\right\rangle\left\langle g_{m^{\prime}}, g_{m}\right\rangle, \\
& \frac{\mathrm{d}^{2}}{\mathrm{~d} t^{2}} \mathrm{FP}\left(\left\{g_{m}(t)\right\}\right)=4 \operatorname{Re} \sum_{m=1}^{M} \sum_{m^{\prime}=1}^{M}\left\{\left\langle g_{m}^{\prime \prime}, g_{m^{\prime}}\right\rangle\left\langle g_{m^{\prime}}, g_{m}\right\rangle+\left\langle g_{m}^{\prime}, g_{m^{\prime}}^{\prime}\right\rangle\left\langle g_{m^{\prime}}, g_{m}\right\rangle\right. \\
&\left.+\left\langle g_{m}^{\prime}, g_{m^{\prime}}\right\rangle\left\langle g_{m^{\prime}}^{\prime}, g_{m}\right\rangle+\left\langle g_{m}^{\prime}, g_{m^{\prime}}\right\rangle\left\langle g_{m^{\prime}}, g_{m}^{\prime}\right\rangle\right\} .
\end{aligned}
$$

Meanwhile, for any $m=1, \ldots, M$,

$$
g_{m}(0)=f_{m}, g_{m}^{\prime}(0)=\left\{\begin{array}{ll}
z_{m} a_{m} h, & m \in \mathcal{I}_{j}, \\
0, & m \notin \mathcal{I}_{j},
\end{array}, g_{m}^{\prime \prime}(0)= \begin{cases}-\left|z_{m}\right|^{2} f_{m}, & m \in \mathcal{I}_{j}, \\
0, & m \notin \mathcal{I}_{j}\end{cases}\right.
$$

Thus, the first-order Taylor coefficient is,

$$
\begin{aligned}
& \left.\frac{\mathrm{d}}{\mathrm{d} t} \mathrm{FP}\left(\left\{g_{m}(t)\right\}\right)\right|_{t=0}=4 \operatorname{Re} \sum_{m \in \mathcal{I}_{j}} \sum_{m^{\prime}=1}^{M}\left\langle z_{m} a_{m} h, f_{m^{\prime}}\right\rangle\left\langle f_{m^{\prime}}, f_{m}\right\rangle \\
& =4 \operatorname{Re} \sum_{m \in \mathcal{I}_{j}}\left\langle F^{*} F z_{m} a_{m} h, f_{m}\right\rangle=4 \lambda_{J} \operatorname{Re} \sum_{m \in \mathcal{I}_{j}} z_{m} a_{m}\left\langle h, f_{m}\right\rangle=0
\end{aligned}
$$

where $\left\langle h, f_{m}\right\rangle=0$ for all $m \in \mathcal{I}_{j}$, as $j \neq J$. As the first-order coefficient is zero, we compute the second-order coefficient,

$$
\begin{array}{r}
\left.\frac{1}{2} \frac{\mathrm{d}^{2}}{\mathrm{~d} t^{2}} \operatorname{FP}\left(\left\{g_{m}(t)\right\}\right)\right|_{t=0}=2 \operatorname{Re}\left\{\sum_{m \in \mathcal{I}_{j}} \sum_{m^{\prime}=1}^{M}\left\langle-\left|z_{m}\right|^{2} f_{m}, f_{m^{\prime}}\right\rangle\left\langle f_{m^{\prime}}, f_{m}\right\rangle\right. \\
+\sum_{m \in \mathcal{I}_{j}} \sum_{m^{\prime} \in \mathcal{I}_{j}}\left[\left\langle z_{m} a_{m} h, z_{m^{\prime}} a_{m^{\prime}} h\right\rangle\left\langle f_{m^{\prime}}, f_{m}\right\rangle+\left\langle z_{m} a_{m} h, f_{m^{\prime}}\right\rangle\left\langle z_{m^{\prime}} a_{m^{\prime}} h, f_{m}\right\rangle\right] \\
\left.+\sum_{m \in \mathcal{I}_{j}} \sum_{m^{\prime}=1}^{M}\left\langle z_{m} a_{m} h, f_{m^{\prime}}\right\rangle\left\langle f_{m^{\prime}}, z_{m} a_{m} h\right\rangle\right\} .
\end{array}
$$

To begin simplifying this expression, note that,

$$
\begin{aligned}
\sum_{m \in \mathcal{I}_{j}} \sum_{m^{\prime}=1}^{M}\left\langle-\left|z_{m}\right|^{2} f_{m}, f_{m^{\prime}}\right\rangle\left\langle f_{m^{\prime}}, f_{m}\right\rangle=-\sum_{m \in \mathcal{I}_{j}}\left|z_{m}\right|^{2}\left\langle F^{*} F f_{m}, f_{m}\right\rangle \\
=-\sum_{m \in \mathcal{I}_{j}}\left|z_{m}\right|^{2} \lambda_{j}\left\langle f_{m}, f_{m}\right\rangle=-\lambda_{j} \sum_{m \in \mathcal{I}_{j}}\left|z_{m}\right|^{2} a_{m}^{2} .
\end{aligned}
$$

Next, the definition of $\left\{z_{m}\right\}$ and the fact that $\left\langle h, f_{m}\right\rangle=0$ for $m \in \mathcal{I}_{j}$ gives, 


$$
\begin{aligned}
& \sum_{m \in \mathcal{I}_{j}} \sum_{m^{\prime} \in \mathcal{I}_{j}}\left[\left\langle z_{m} a_{m} h, z_{m^{\prime}} a_{m^{\prime}} h\right\rangle\left\langle f_{m^{\prime}}, f_{m}\right\rangle+\left\langle z_{m} a_{m} h, f_{m^{\prime}}\right\rangle\left\langle z_{m^{\prime}} a_{m^{\prime}} h, f_{m}\right\rangle\right] \\
= & \sum_{m \in \mathcal{I}_{j}} \sum_{m^{\prime} \in \mathcal{I}_{j}}\|h\|^{2}\left\langle\overline{z_{m^{\prime}}} a_{m^{\prime}} f_{m^{\prime}}, \overline{z_{m}} a_{m} f_{m}\right\rangle=\left\|\sum_{m \in \mathcal{I}_{j}} \overline{z_{m}} a_{m} f_{m}\right\|^{2}=\|0\|^{2}=0 .
\end{aligned}
$$

Finally,

$$
\begin{aligned}
& \sum_{m \in \mathcal{I}_{j}} \sum_{m^{\prime}=1}^{M}\left\langle z_{m} a_{m} h, f_{m^{\prime}}\right\rangle\left\langle f_{m^{\prime}}, z_{m} a_{m} h\right\rangle=\sum_{m \in \mathcal{I}_{j}}\left|z_{m}\right|^{2} a_{m}^{2}\left\langle F^{*} F h, h\right\rangle \\
&=\sum_{m \in \mathcal{I}_{j}}\left|z_{m}\right|^{2} a_{m}^{2} \lambda_{J}\langle h, h\rangle=\lambda_{J} \sum_{m \in \mathcal{I}_{j}}\left|z_{m}\right|^{2} a_{m}^{2} .
\end{aligned}
$$

Thus, the second-order Taylor coefficient is,

$$
\left.\frac{1}{2} \frac{\mathrm{d}^{2}}{\mathrm{~d} t^{2}} \mathrm{FP}\left(\left\{g_{m}(t)\right\}\right)\right|_{t=0}=2\left(\lambda_{j}-\lambda_{J}\right) \sum_{m \in \mathcal{I}_{j}}\left|z_{m}\right|^{2} a_{m}^{2} .
$$

To determine the sign of this coefficient, note that $\lambda_{j}>\lambda_{J}$ since $j<J$. Also, the sequence $\left\{z_{m}\right\}_{m=1}^{M}$ is nonzero, by assumption. Furthermore, since $\mathcal{I}_{J}$ contains the indices of any zero elements of $\left\{f_{m}\right\}_{m=1}^{M}$ by decree, then $a_{m}=\left\|f_{m}\right\|>0$ for all $m \in \mathcal{I}_{j}$. Thus, the second-order coefficient is negative. An explicit, straightforward computation reveals that the third derivative of $\operatorname{FP}\left(\left\{g_{m}(t)\right\}\right)$ is bounded near zero. Thus, by Taylor's theorem, $\operatorname{FP}\left(\left\{g_{m}(t)\right\}_{m=1}^{M}\right)<\operatorname{FP}\left(\left\{f_{m}\right\}_{m=1}^{M}\right)$ for all sufficiently small $t$, a contradiction.

Claim 4: For any $j<J,\left(1 / \sqrt{\lambda_{j}}\right)\left\{f_{m}\right\}_{m \in \mathcal{I}_{j}}$ is an orthonormal basis for $E_{j}$. First note that since $j<J$, then $\lambda_{j}>\lambda_{J} \geq 0$. This, combined with the previous two claims, gives that $\left\{f_{m}\right\}_{m \in \mathcal{I}_{j}}$ is a linearly independent $\lambda_{j}$-tight frame for $E_{j}$. As a tight frame is necessarily a spanning set, $\left\{f_{m}\right\}_{m \in \mathcal{I}_{j}}$ is a basis for $E_{j}$. Thus, $\left|\mathcal{I}_{j}\right|=\operatorname{dim} E_{j}$, and so the analysis operator $F_{j}: E_{j} \rightarrow \mathrm{C}^{\left|\mathcal{I}_{j}\right|}$ which already satisfies $F_{j}^{*} F_{j}=\lambda_{j} I$ must also satisfy $F_{j} F_{j}^{*}=\lambda_{j} I$. Letting $\left\{e_{m}\right\}_{m \in \mathcal{I}_{J}}$ be the standard basis for $\mathrm{C}^{\left|\mathcal{I}_{j}\right|}$, we have that for any $m, m^{\prime} \in \mathcal{I}_{j}$,

$$
\left\langle f_{m}, f_{m^{\prime}}\right\rangle=\left\langle F_{j}^{*} e_{m}, F_{j}^{*} e_{m^{\prime}}\right\rangle=\left\langle F_{j} F_{j}^{*} e_{m}, e_{m^{\prime}}\right\rangle=\lambda_{j}\left\langle e_{m}, e_{m^{\prime}}\right\rangle .
$$

Thus, $\left\{f_{m}\right\}_{m \in \mathcal{I}_{j}}$ is orthogonal and $a_{m}^{2}=\left\|f_{m}\right\|^{2}=\lambda_{j}$ for all $m \in \mathcal{I}_{j}$.

Claim 5: $\left(N-M+\left|\mathcal{I}_{J}\right|\right) \lambda_{J}=\sum_{m \in \mathcal{I}_{J}} a_{m}^{2}$. For any $j<J$, the previous claim gives that $\left\{f_{m}\right\}_{m \in \mathcal{I}_{j}}$ is a basis for $E_{j}$. Thus,

$$
M=\sum_{j=1}^{J}\left|\mathcal{I}_{j}\right|=\left|\mathcal{I}_{J}\right|+\sum_{j=1}^{J-1}\left|\mathcal{I}_{j}\right|=\left|\mathcal{I}_{J}\right|+\sum_{j=1}^{J-1} \operatorname{dim} E_{j}=\left|\mathcal{I}_{J}\right|+N-\operatorname{dim} E_{J}
$$

By the second claim, $\left\{f_{m}\right\}_{m \in \mathcal{I}_{J}}$ is $\lambda_{J}$-tight for $E_{J}$. Thus, by Proposition 1 , 


$$
\left(N-M+\left|\mathcal{I}_{J}\right|\right) \lambda_{J}=\left(\operatorname{dim} E_{J}\right) \lambda_{J}=\sum_{m \in \mathcal{I}_{J}} a_{m}^{2} .
$$

Claim 6: $\left\{N_{0}, \ldots, M\right\} \subseteq \mathcal{I}_{J}$. We prove the complement inclusion, namely $\mathcal{I}_{J}^{C} \subseteq\left\{1, \ldots, N_{0}-1\right\}$. Take any $n \in \mathcal{I}_{j} \subset \mathcal{I}_{J}^{C}$. Using the previous claim and the fact that $\left\{a_{m}^{2}\right\}_{m=1}^{M}$ is decreasing, note that,

$$
\begin{aligned}
\sum_{m=n+1}^{M} a_{m}^{2} & =\left|\mathcal{I}_{J} \cap\{1, \ldots, n\}\right| a_{n}^{2}+\sum_{m=n+1}^{M} a_{m}^{2}-\left|\mathcal{I}_{J} \cap\{1, \ldots, n\}\right| a_{n}^{2}, \\
& \leq \sum_{m \in \mathcal{I}_{J}, m=1}^{n} a_{m}^{2}+\sum_{m=n+1}^{M} a_{m}^{2}-\left|\mathcal{I}_{J} \cap\{1, \ldots, n\}\right| a_{n}^{2}, \\
& =\sum_{m \in \mathcal{I}_{J}} a_{m}^{2}+\sum_{m \in \mathcal{I}_{J}^{C}, m=n+1}^{M} a_{m}^{2}-\left|\mathcal{I}_{J} \cap\{1, \ldots, n\}\right| a_{n}^{2}, \\
& <\left[N-M+\left|\mathcal{I}_{J}\right|+\left|\mathcal{I}_{J}^{C} \cap\{n+1, \ldots, M\}\right|-\left|\mathcal{I}_{J} \cap\{1, \ldots, n\}\right|\right] a_{n}^{2}, \\
& =[N-M+|\{n+1, \ldots, N\}|] a_{n}^{2}=(N-n) a_{n}^{2} .
\end{aligned}
$$

As defined in Lemma 2, the index $N_{0}$ is the starting point at which the opposite inequality begins to hold. Thus, $n<N_{0}$, that is, $n \in\left\{0, \ldots, N_{0}-1\right\}$.

Claim 7: $\left\{N_{0}, \ldots, M\right\}=\mathcal{I}_{J}$. By the previous claim, it suffices to show $\left\{1, \ldots, N_{0}-1\right\} \cap \mathcal{I}_{J}=\emptyset$. Assume to the contrary that $\left\{1, \ldots, N_{0}-1\right\} \cap \mathcal{I}_{J} \neq \emptyset$, with minimal index $n_{0}$ and maximal index $n_{1}$. Applying Proposition 1 along with the second and fifth claims gives,

$$
\left[N-M+\left|\mathcal{I}_{J}\right|-1\right] a_{n_{0}}^{2} \leq\left[N-M+\left|\mathcal{I}_{J}\right|\right] \lambda_{J}-a_{n_{0}}^{2} \leq \sum_{m \in \mathcal{I}_{J}, m>n_{0}} a_{m}^{2} .
$$

When applied to the sequence $\left\{a_{m}^{2}\right\}_{m \in \mathcal{I}_{J}}$, Lemma 2 then guarantees a similar inequality holds at $n_{1}$,

$$
\left[N-M+\left|\mathcal{I}_{J}\right|-\left|\left\{1, \ldots, n_{1}\right\} \cap \mathcal{I}_{J}\right|\right] a_{n_{1}}^{2} \leq \sum_{m \in \mathcal{I}_{J}, m>n_{1}} a_{m}^{2} .
$$

By the previous claim and the definition of $n_{1}$, this reduces to,

$$
\left[N-\left(N_{0}-1\right)\right] a_{N_{0}-1}^{2} \leq\left[N-M+\left|\left\{N_{0}, \ldots, M\right\}\right|\right] a_{n_{1}}^{2} \leq \sum_{m=N_{0}}^{M} a_{m}^{2} .
$$

However, the definition of the irregularity $N_{0}$ gives that,

$$
(N-n) a_{n}^{2}>\sum_{m=n+1}^{M} a_{m}^{2},
$$

for all $n<N_{0}$, which is a contradiction when $n=N_{0}-1$. 
To complete the proof we need only summarize our progress. By the first and seventh claim,

$$
\left\{f_{m}\right\}_{m=1}^{M}=\left\{f_{m}\right\}_{m \in \mathcal{I}_{J}^{C}} \cup\left\{f_{m}\right\}_{m \in \mathcal{I}_{J}}=\left\{f_{m}\right\}_{m=1}^{N_{0}-1} \cup\left\{f_{m}\right\}_{N_{0}}^{M},
$$

where, by the fourth claim, $\left\{f_{m}\right\}_{m=1}^{N_{0}-1}$ is an orthogonal basis for $\operatorname{Span}\left\{E_{j}\right\}_{j=1}^{J-1}$, and, by the second claim, $\left\{f_{m}\right\}_{m=N_{0}}^{M}$ is tight for $E_{J}$, which is the orthogonal complement of $\operatorname{Span}\left\{f_{m}\right\}_{m=N_{0}}^{M}=\operatorname{Span}\left\{E_{j}\right\}_{j=1}^{J-1}$. Finally, in the case where the number of nonzero elements of $\left\{a_{m}\right\}_{m=1}^{M}$ is at least $N$, then at least one element of $\left\{f_{m}\right\}_{m=N_{0}}^{M}$ is nonzero. By Proposition 1, the corresponding tightness constant is then positive, and so $\left\{f_{m}\right\}_{m=N_{0}}^{M}$ is a tight frame.

We note that for the special case of $a_{m}=1$ for all $m$, this result reduces to the main result of Benedetto and Fickus [2]. As a consequence of Theorem 1, we next demonstrate the sufficiency of the fundamental inequality, answering the question that began our chapter.

Corollary 1. Let $\mathbb{H}_{N}$ be an $N$-dimensional Hilbert space, and let $\left\{a_{m}\right\}_{m=1}^{M}$ be a nonzero nonnegative sequence. Then, there exists a tight frame $\left\{f_{m}\right\}_{m=1}^{M}$ for $\mathbb{H}_{N}$ with $\left\|f_{m}\right\|=a_{m}$ for all $m$ if and only if $\left\{a_{m}\right\}_{m=1}^{M}$ satisfies the fundamental inequality.

Proof. The necessity of the fundamental inequality is given by Proposition 1 . For the converse, let $\left\{a_{m}\right\}_{m=1}^{M}$ satisfy the fundamental inequality. By Lemma 3 , the regularity $N_{0}-1$ of $\left\{a_{m}\right\}_{m=1}^{M}$ is zero. Next, note that the frame potential is clearly continuous over the compact set $\mathrm{S}\left(\left\{a_{m}\right\}_{m=1}^{M}\right)$, and thus has a global minimizer $\left\{f_{m}\right\} \in \mathrm{S}\left(\left\{a_{m}\right\}_{m=1}^{M}\right)$. As global minimizers are necessarily local, Theorem 1 gives that $\left\{f_{m}\right\}_{m=1}^{M}$ is of the form,

$$
\left\{f_{m}\right\}_{m=1}^{M}=\left\{f_{m}\right\}_{m=1}^{0} \cup\left\{f_{m}\right\}_{m=1}^{M},
$$

where $\left\{f_{m}\right\}_{m=1}^{M}$ is tight for the orthogonal complement of $\left\{f_{m}\right\}_{m=1}^{0}=\emptyset$, namely $\mathbb{H}_{N}$. By combining Proposition 1 with the fact that $\left\{a_{m}\right\}_{m=1}^{M}$ is nonzero, we conclude the tightness constant is positive.

As another corollary of Theorem 1, we improve upon Proposition 4 by finding the actual minimum value of the frame potential when the fundamental inequality is violated.

Corollary 2. Let $\mathbb{H}_{N}$ be any $N$-dimensional Hilbert space, and let $\left\{a_{m}\right\}_{m=1}^{M}$ be a nonnegative decreasing sequence of irregularity $N_{0}-1$. Then, any local minimizer of the frame potential $\mathrm{FP}: \mathrm{S}\left(\left\{a_{m}\right\}_{m=1}^{M}\right) \rightarrow \mathbb{R}$ is also a global minimizer, and the minimum value is,

$$
\sum_{m=1}^{N_{0}-1} a_{m}^{4}+\frac{1}{N-N_{0}+1}\left[\sum_{m=N_{0}}^{M} a_{m}^{2}\right]^{2} .
$$


Proof. First note that the frame potential is continuous over the compact set $\mathrm{S}\left(\left\{a_{m}\right\}_{m=1}^{M}\right)$, and thus has at least one local minimizer. By Theorem 1, any local minimizer of the frame potential consists of an orthogonal sequence $\left\{f_{m}\right\}_{m=1}^{N_{0}-1}$ for whose orthogonal complement the sequence $\left\{f_{m}\right\}_{m=N_{0}}^{M}$ is tight. We compute the frame potential of any such sequence. Since $\left\langle f_{m}, f_{m^{\prime}}\right\rangle=0$ for any $m^{\prime} \neq m=1, \ldots, N_{0}-1$,

$$
\begin{aligned}
\mathrm{FP}\left(\left\{f_{m}\right\}_{m=1}^{M}\right)=\sum_{m=1}^{N_{0}-1}\left|\left\langle f_{m}, f_{m}\right\rangle\right|^{2}+\sum_{m=N_{0}}^{M} \sum_{m^{\prime}=N_{0}}^{M}\left|\left\langle f_{m}, f_{m^{\prime}}\right\rangle\right|^{2} & \\
& =\sum_{m=1}^{N_{0}-1} a_{m}^{4}+\operatorname{FP}\left(\left\{f_{m}\right\}_{m=N_{0}}^{M}\right) .
\end{aligned}
$$

Note that the definition of the irregularity $N_{0}$ implies that the radii $\left\{a_{m}\right\}_{m=1}^{N_{0}}$ are strictly positive. Thus, the dimension of the span of $\left\{f_{m}\right\}_{m=1}^{N_{0}-1}$ is $N_{0}-1$, and so the dimension of the orthogonal complement is $\left(N-N_{0}+1\right)$. As $\left\{f_{m}\right\}_{m=N_{0}}^{M}$ is tight for this space, the frame potential of $\left\{f_{m}\right\}_{m=N_{0}}^{M}$ is given by Proposition 4, yielding,

$$
\operatorname{FP}\left(\left\{f_{m}\right\}_{m=1}^{M}\right)=\sum_{m=1}^{N_{0}-1} a_{m}^{4}+\frac{1}{N-N_{0}+1}\left[\sum_{m=N_{0}}^{M} a_{m}^{2}\right]^{2}
$$

To summarize, there exists at least one local minimizer of the frame potential, and all local minimizers attain the same value (8). Thus, every local minimizer is also a global minimizer, and the minimum value is (8).

We now interpret these results in the context of the physical theory. Corollary 2 is surprising from this point of view. In particular, consider the classical problem of minimizing the potential energy,

$$
\sum_{m=1}^{M} \sum_{m^{\prime} \neq m} \frac{1}{\left\|f_{m}-f_{m^{\prime}}\right\|}
$$

of a system of $M$ electrons on a conductive spherical shell which exhibit a force upon one another in accordance with Coulomb's Law. For all but the smallest numbers of points, the function (9) has many local minimizers which are not global. For example, a dodecahedral arrangement of twenty points is known to be in equilibrium under Coulomb's Law but not optimal.

The physical theory is especially helpful in gaining an intuitive understanding of the fundamental inequality. As before, consider a dynamic physical system of $M$ points $\left\{f_{m}\right\}_{m=1}^{M}$ in which each point exerts a frame force upon all the others, and in which the movement of the points is restricted to concentric spheres about the origin of radii $\left\{a_{m}\right\}_{m=1}^{M}$.

Though each point wants to make the others perpendicular to itself, this is clearly impossible if the number of nonzero radii $a_{m}$ is greater than the 
dimension $N$. At the same time, recall that a point a certain distance from the origin gives off a greater push than other, closer points. Specifically, recall that the power of the frame force field is taken to be $a_{m}^{2}$. Thus, when a single radius $a_{m^{\prime}}$ is disproportionately large, it is conceivable that $f_{m^{\prime}}$ may be strong enough to force all the other points into an orthogonal hyperplane.

Remarkably, Theorem 1 not only verifies that this phenomenon actually occurs, it also provides a quantitative way to determine the degree to which it happens. For example, consider a decreasing sequence $\left\{a_{m}\right\}_{m=1}^{M}$ whose irregularity $N_{0}-1$ is at least one. Let $\left\{f_{m}\right\} \in \mathrm{S}\left(\left\{a_{m}\right\}_{m=1}^{M}\right)$ be a local minimizer of the frame potential. Here, the definition of irregularity gives,

$$
a_{1}^{2}>\frac{1}{N-1} \sum_{m=2}^{M} a_{m}^{2} .
$$

At the same time, Theorem 1 guarantees that $f_{1}$ is necessarily orthogonal to $\left\{f_{m}\right\}_{m=2}^{M}$. Thus, as long as $f_{1}$ is stronger than the "dimensional average" of the remaining points, then $f_{1}$ is powerful enough to take an entire dimension for itself, leaving the other points to fight over the remaining $N-1$ dimensions.

These points then repeat the above scenario on a smaller scale. In particular, if the irregularity of the original sequence is at least two, then,

$$
a_{2}^{2}>\frac{1}{N-2} \sum_{m=3}^{M} a_{m}^{2} .
$$

Here, $f_{2}$ takes its fill, and lets $\left\{f_{m}\right\}_{m=3}^{M}$ fight over the $(N-2)$-dimensional leftovers. This process keeps repeating until eventually, perhaps at $N_{0}=N$, we move beyond the point of irregularity. Here, none of the remaining points $\left\{f_{m}\right\}_{m=N_{0}}^{M}$ are strong enough to overcome the others. Forced to share the orthogonal complement of $\left\{f_{m}\right\}_{m=1}^{N_{0}}$, these points eventually settle into a tight equilibrium. In the special case when the fundamental inequality is satisfied, this balance is achieved from the very beginning, creating a tight frame for the entire space.

As an example of these phenomena, consider the following parametrized family of tight frames of three elements for $\mathbb{R}^{2}$,

$$
\left\{\left[\begin{array}{r}
0 \\
\sqrt{-2 \cos 2 t}
\end{array}\right],\left[\begin{array}{l}
\sin t \\
\cos t
\end{array}\right],\left[\begin{array}{r}
-\sin t \\
\cos t
\end{array}\right]\right\}
$$

where $t \in[\pi / 2,2 \pi / 3]$. When $t=2 \pi / 3$, this becomes the so-called MercedesBenz UNTF, as pictured on the far left of Figure 1. As $t$ gets smaller, the first element moves away from the origin, while the others only change orientation. In particular, the field generated by the first element grows stronger, forcing the other two to become increasingly perpendicular in order to preserve the equilibrium, as pictured in the second image from the left in Figure 1. 
However, this process does not continue forever. In particular at $t=\pi / 2$, the lengths of the frame elements are $\{\sqrt{2}, 1,1\}$, and the fundamental inequality is satisfied as an equality. As pictured in the second image from the right in Figure 1, the resulting arrangement is still tight, but just barely. Beyond this parameter, an increase in the length of the first element may no longer be compensated for by the other two points; it is not possible to be "more orthogonal" than already being orthogonal. The resulting arrangements, as seen on the far right of Figure 1, are still optimal for that set of given lengths, but the balance is gone, and the tightness is lost.

Fig. 1. A paramterized family of local minimizers of the frame potential.
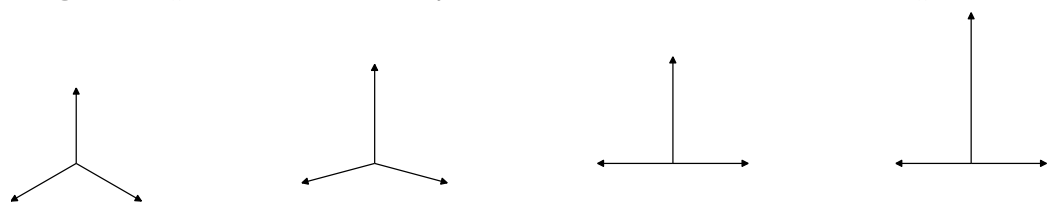

We conclude this chapter with a brief discussion of Viswanath and Anantharam's discovery of the fundamental inequality during their investigation of the capacity region in synchronous Code-Division Multiple Access (CDMA) systems. In a CDMA system, there are $M$ users who share the available spectrum. The sharing is achieved by "scrambling" $M$-dimensional user vectors into smaller, $N$-dimensional vectors. In terms of frame theory, this scrambling corresponds to the application of a synthesis operator $S=F^{*}$ corresponding to $M$ distinct $N$-dimensional signature vectors of length $\sqrt{N}$. Noise-corrupted versions of these synthesized vectors arrive at a receiver, where the signature vectors are used to help extract the original user vectors.

Viswanath and Anantharam showed that the design of the optimal signature matrix $S$ depends upon the powers $\left\{p_{m}\right\}_{m=1}^{M}$ of the individual users. In particular, they divided the users into two classes: those that are oversized and those that are not, by applying the idea of Lemma 2 to $\left\{p_{m}\right\}_{m=1}^{M}$. While the oversized users are assigned orthogonal channels for their personal use, the remaining users have their signature vectors designed so as to be Welch Bound Equality (WBE) sequences, namely, sequences which achieve the lower bound of Proposition 4, which are necessarily tight frames.

When no user is oversized, that is, when the fundamental inequality is satisfied, Viswanath and Anantharam show that the optimal signature sequences $S$ must satisfy $S D S^{*}=p_{\text {tot }} I$, where $D$ is a diagonal matrix whose entries are the powers $\left\{p_{m}\right\}_{m=1}^{M}$, and where $p_{\text {tot }}=\sum_{m=1}^{M} p_{m}$. By letting $F=D^{1 / 2} S^{*} / \sqrt{N}$, this problem reduces to finding an $M \times N$ matrix $F$ whose $m$ th row is of norm $\sqrt{p_{m}}$, and such that $F^{*} F=\left(p_{\text {tot }} / N\right) I$. That is, their problem reduces to finding a tight frame for $\mathbb{H}_{N}$ of lengths $\left\{\sqrt{p_{m}}\right\}_{m=1}^{M}$. While Viswanath and Anantharam gave one solution to this problem using an ex- 
plicit construction, we have characterized all solutions to this problem using a physical interpretation of frame theory.

\section{References}

1. J.J. Benedetto and D. Colella. Wavelet analysis of spectogram seizure chips. In Proc. SPIE Conf. on Wavelet Appl. in Signal and Image Proc., pages 512-521, San Diego, CA, July 1995.

2. J.J. Benedetto and M. Fickus. Finite normalized tight frames. Advances in Computational Mathematics, 18(2-4):357-385, February 2003

3. J.J. Benedetto and G.E. Pfander. Wavelet periodicity detection algorithms. In Proc. SPIE Conf. on Wavelet Appl. in Signal and Image Proc., pages 48-55, San Diego, CA, July 1998.

4. H. Bolcskei, F. Hlawatsch, and H.G. Feichtinger. Frame-theoretic analysis of oversampled filter banks. IEEE Trans. Signal Proc., 46(12):3256-3269, December 1998 .

5. P.G. Casazza and J. Kovačević. Equal-norm tight frames with erasures. Advances in Computational Mathematics, 18(2-4):387-430, February 2003.

6. Z. Cvetković. Resilience properties of redundant expansions under additive noise and quantization. IEEE Trans. Inform. Th., 49(3):644-656, March 2003. To appear.

7. Z. Cvetković and M. Vetterli. Oversampled filter banks. Signal Proc., 46(5):1245-1255, May 1998.

8. I. Daubechies. Ten Lectures on Wavelets. SIAM, Philadelphia, PA, 1992.

9. I. Daubechies, A. Grossman, and Y. Meyer. Painless nonorthogonal expansions. Journ. Math. Phys., 27:1271-1283, November 1986.

10. R. J. Duffin and A. C. Schaeffer. A class of nonharmonic Fourier series. Trans. Amer. Math. Soc., 72:341-366, 1952.

11. K. Dykema, D. Freeman, K. Kornelson, D. Larson, M. Ordower and E. Weber. Ellipsoidal Tight Frames and Projection Decomposition of Operators, Illinois Journal of Mathematics, 48(2):477-489, Summer 2004.

12. K. Dykema and N. Strawn. Manifold Structure of Spaces of Spherical Tight Frames (Preprint).

13. D. Gabor. Theory of communication. Journ. IEE, 93:429-457, 1946.

14. V.K Goyal, J. Kovačević and J.A. Kelner. Quantized frame expansions with erasures. Journal of Appl. and Comput. Harmonic Analysis, 10(3):203-233, May 2001.

15. V. K Goyal, M. Vetterli, and N. T. Thao. Quantized overcomplete expansions in $\mathbb{R}^{N}$ : Analysis, synthesis, and algorithms. IEEE Trans. Inform. Th., 44(1):16-31, January 1998.

16. B. Hochwald, T. Marzetta, T. Richardson, W. Sweldens, and R. Urbanke. Systematic design of unitary space-time constellations. IEEE Trans. Info. Th., vol. 46, pp.1962-1973, September 2000.

17. R. Holmes and V. Paulsen. Optimal Frames for Erasures. Lin. Alg. Appl.. 377:31-51, January 2004.

18. J. Kovačević, P.L. Dragotti, and V.K Goyal. Filter bank frame expansions with erasures. IEEE Trans. Inform. Th., special issue in Honor of Aaron D. Wyner, 48(6):1439-1450, June 2002. Invited paper. 
19. T. Strohmer. Modern Sampling Theory: Mathematics and Applications, chapter Finite and infinite-dimensional models for oversampled filter banks, pages 297320. Birkhauser, Boston, 2000.

20. T. Strohmer and R. Heath, Jr. Grassmannian frames with applications to coding and communications. Appl. Comp. Harm. Anal., vol.14(3): 257-275, 2003.

21. M. Unser. Texture classification and segmentation using wavelet frames. IEEE Trans. Image Proc., 4:1549-1560, November 1995.

22. R. Vale and S. Waldron. Tight frames and their symmetries. Const. Approx. (to appear).

23. P. Viswanath and V. Anantharam, Optimal Sequences and Sum Capacity of Synchronous CDMA Systems. IEEE Transactions on Information Theory, Sept. 1999, Vol.45, No.6, pp. 1984-1991.

24. S. Waldron. Generalised Welch Bound Equality sequences are tight frames. IEEE Trans. Info. Th., vol. 49, no. 9, pp. 2307-2309, September 2003. 